REGARDS

SUR L'ECONOMIE ALLEMAND

BULLETIN ECONOMIQUE DU CIRAC
Regards sur l'économie allemande

Bulletin économique du CIRAC

$97 \mid 2010$

Varia

\title{
Grandes Villes : elles grandissent et rajeunissent
}

Isabelle Bourgeois

\section{CpenEdition}

\section{Journals}

Édition électronique

URL : http://journals.openedition.org/rea/4145

DOI : $10.4000 /$ rea.4145

ISBN : 978-2-8218-0890-4

ISSN : 1965-0787

Éditeur

CIRAC

Édition imprimée

Date de publication : 1 juillet 2010

Pagination : 33-34

ISSN : 1156-8992

Référence électronique

Isabelle Bourgeois, "Grandes Villes : elles grandissent et rajeunissent », Regards sur l'économie allemande [En ligne], 97 | juillet 2010, mis en ligne le 13 juillet 2010, consulté le 15 septembre 2020. URL : http://journals.openedition.org/rea/4145

Ce document a été généré automatiquement le 15 septembre 2020

(C) CIRAC 


\title{
Grandes Villes : elles grandissent et rajeunissent
}

\author{
Isabelle Bourgeois
}

1 Les villes allemandes ont gagné en attractivité, voyant leur population croître de près de $3 \%$ entre 1999 et 2008 ; dans le même temps, la population allemande reculait. Et alors que globalement l'emploi stagnait outre-Rhin, il augmentait de $4 \%$ en milieu urbain. Alors que la part des plus âgés décroît, c'est chez les 25-30 ans que l'évolution est la plus nette : cette population a augmenté de plus de $12 \%$, alors qu'elle reculait de près de $5 \%$ dans l'ensemble de l'Allemagne. Les villes allemandes attirent de plus en plus de jeunes hautement qualifiés et avec une rémunération afférente, révèle une étude de l'institut DIW (DIW-Wochenbericht 19/2010) qui se consacre à la quinzaine de villes de plus de 500000 habitants que compte l'Allemagne,

\section{Munich, Dresde et Leipzig sont les plus dynamiques}

2 C'est Munich qui est de loin la plus dynamique (avec une hausse de plus de $11 \%$ de sa population), suivie de Dresde ( $+7 \%)$ et Leipzig $(+5 \%)$. Berlin et Brême ( $+1,5 \%$ chacune) sont à la traîne, alors que Dortmund, Duisburg et Essen ont perdu des habitants. Ce sont aussi Leipzig, Dresde et Munich qui ont attiré le plus de femmes de $25-30$ ans (+40\%), suivies par Francfort/Main, Berlin, Cologne et Hambourg (plus de $15 \%$ dans chaque cas).

3 Si les villes rajeunissent, c'est que les jeunes déjà qualifiés y trouvent une offre de formation qui les séduit, notamment celle des grandes universités. Ce qui les incite à rester et à s'installer dans le centre-ville, c'est la richesse de l'offre culturelle et de loisirs, mais aussi l'existence d'un marché locatif de plus en plus à leur mesure. Quant aux jeunes femmes, elles y trouvent aussi les infrastructures nécessaires pour concilier études/vie professionnelle et vie familiale. 


\section{Concentration des services intensifs en savoir}

4 Certes, les villes ont perdu beaucoup plus d'emplois industriels que l'ensemble de l'Allemagne (en moyenne, cette part est de 12,6\% aujourd'hui dans les centres urbains), mais ce recul est largement compensé par tous les services autres que de proximité et fortement internationalisés, qui représentent désormais $19 \%$ des activités dans les villes. Plus particulièrement, ce sont les services intensifs en savoir et liés aux entreprises qui tirent l'économie urbaine ; leur part a augmenté de $23 \%$ (contre $15 \%$ seulement en moyenne allemande).

\section{Les entreprises se localisent près des gisements de compétences}

5 Ces phénomènes sont la résultante de deux tendances co-existantes qui ont tout de la poule et de l'œuf: les activités intensives en savoir exigent des entreprises qu'elles constituent des grappes et misent sur la culture d'échanges et de réseaux que présentent les agglomérations; les "têtes hautement qualifiées" (DIW) sont, elles, en quête d'un lifestyle urbain. Or "comme les créatifs veulent vivre en ville et que l'économie du savoir a besoin de leur savoir-faire, les entreprises suivent les désirs de localisation de la ressource humaine». Si ces évolutions présentent des avantages en termes de compétitivité économique, elles ont néanmoins un revers : le risque de voir petit à petit exclure du centre-ville les moins aisés et les moins qualifiés. Un risque que les auteurs de l'étude conseillent vivement aux urbanistes de mieux prendre en considération dans leurs projets notamment pour veiller à préserver la mixité sociale du logement qui reste une caractéristique des grandes villes allemandes.

\section{INDEX}

Mots-clés : commune, ville, urbanisme, urbanisation, démographie, entreprise, aménagement du territoire 